PROCEEDINGS OF THE

AMERICAN MATHEMATICAL SOCIETY

Volume 139, Number 5, May 2011, Pages 1851-1863

S 0002-9939(2010)10614-7

Article electronically published on October 20, 2010

\title{
ON THE YANG-MILLS STRATIFICATION FOR SURFACES
}

\author{
DANIEL A. RAMRAS
}

(Communicated by Richard A. Wentworth)

\begin{abstract}
Atiyah and Bott showed that Morse theory for the Yang-Mills functional can be used to study the space of flat, or more generally central, connections on a bundle over a Riemann surface. These methods have recently been extended to non-orientable surfaces by Ho and Liu. In this article, we use Morse theory to determine the exact connectivity of the natural map from the homotopy orbits of the space of central Yang-Mills connections to the classifying space of the gauge group. The key ingredient in this computation is a combinatorial study of the Morse indices of Yang-Mills critical sets.
\end{abstract}

\section{INTRODUCTION}

Let $M^{g}$ be a Riemann surface of genus $g>0$, and consider a vector bundle $E$ over $M^{g}$. When $E$ is trivial, the space $\mathcal{A}_{\text {flat }}(E)$ of flat connections on $E$ forms the minimum critical set for the Yang-Mills functional $L: \mathcal{A}(E) \rightarrow \mathbb{R}$, where $\mathcal{A}(E)$ is the affine space of all connections, and for $A \in \mathcal{A}(E)$,

$$
L(A)=\int_{M}\|F(A)\|^{2} d \mathrm{vol}_{M}
$$

Here $F(A)$ is the curvature form of $A$, and the volume of $M$ is normalized to 1 . In their seminal paper on Yang-Mills theory [1, Atiyah and Bott showed that by treating $L$ as a gauge-equivariant Morse function, one can learn a great deal about the topology of the critical set $\mathcal{A}_{\text {flat }}(E)$ and its stable manifold $\mathcal{C}_{\text {ss }}(E)$, the space of semi-stable holomorphic structures on $E$. In particular, Atiyah and Bott provided a framework for computing the gauge-equivariant cohomology of these spaces.

For a general bundle $E$, the minimum critical set $\mathcal{C}_{\min }(E)$ of $L$ consists of central Yang-Mills connections, and again, the stable manifold of this critical set is $\mathcal{C}_{\mathrm{ss}}(E)$. By work of Daskalopoulos [5] and Råde [13] (relying heavily on Uhlenbeck's compactness theorem [16]), the Yang-Mills flow provides a deformation retraction $\mathcal{C}_{\mathrm{ss}}(E) \stackrel{\widetilde{\sim}}{\rightarrow} \mathcal{C}_{\min }(E)$, and hence we refer to $\mathcal{C}_{\mathrm{ss}}(E)$ as the stable manifold of $\mathcal{C}_{\min }(E)$. The methods of Atiyah and Bott apply directly to $\mathcal{C}_{\mathrm{ss}}(E)$ and then extend to $\mathcal{C}_{\min }(E)$ via this homotopy equivalence.

Recently, Ho and Liu [7] have constructed an analogous Morse Theory for connections on a bundle $E$ over a non-orientable surface $\Sigma$. In this setting, the minimum critical set is always the space of flat connections on $E$. Their methods have led to

Received by the editors January 13, 2010 and, in revised form, May 24, 2010.

2010 Mathematics Subject Classification. Primary 53C07, 58D27; Secondary 58E15, 05A20.

This work was partially supported by NSF grants DMS-0353640 (RTG), DMS-0804553, and DMS-0968766. 
computations of the $U(n)$-equivariant Poincaré series of $\operatorname{Hom}\left(\pi_{1} \Sigma, U(n)\right)$ for small $n$ (see Ho-Liu 9] and Baird [2]).

We now describe our main result, Theorem 3.15. Let $M$ be a surface and consider the map

$$
E \mathcal{G}(E) \times_{\mathcal{G}(E)} \mathcal{C}_{\text {min }}(E)=\mathcal{C}_{\text {min }}(E)_{h \mathcal{G}(E)} \stackrel{q}{\rightarrow} \operatorname{Map}^{E}(M, B U(n))=B \mathcal{G}(E)
$$

from the homotopy orbits of $\mathcal{C}_{\min }(E)$ under the gauge group $\mathcal{G}(E)$ to the classifying space of $\mathcal{G}(E)$. First, consider the case in which $M$ is a Riemann surface of genus $g$ and $E$ has rank $n>1$ and Chern number $k$. Given $r \in \mathbb{Z}$, let $[r]_{n}$ denote the unique integer between 1 and $n$ equivalent to $r$ modulo $n$. Using Yang-Mills theory, we show that if $g>1$, then the connectivity of the map (11) is precisely

$$
2 \min \left([k]_{n},[-k]_{n}\right)+2(g-1)(n-1)-1 .
$$

When $g=1$, we obtain the simpler formula $2 \operatorname{gcd}(n, k)-1$ for this connectivity. On the other hand, say $M$ is non-orientable and the genus $\widetilde{g}$ of its orientable double cover is at least 2 . Then if $E$ has rank $n \geqslant 9$, we show that $q$ is precisely $(2 n \widetilde{g}-3 \widetilde{g})-$ connected. We also obtain formulas when $\widetilde{g}=1$, but they are not as clean.

In (11), one may replace $\mathcal{G}(E)$ by the based gauge group $\mathcal{G}_{0}(E)$ without affecting the connectivity of $q$. If $M$ is orientable and $E$ is trivial, $\mathcal{C}_{\min }(E)=\mathcal{A}_{\text {flat }}(E)$ and we obtain the connectivity of the map

$$
\operatorname{Hom}\left(\pi_{1} M, U(n)\right) \cong \mathcal{A}_{\text {flat }}(E) / \mathcal{G}_{0}(E) \simeq \mathcal{A}_{\text {flat }}(E)_{h \mathcal{G}_{0}(E)} \rightarrow B \mathcal{G}_{0}(E) .
$$

Similarly, if $\Sigma$ is a non-orientable surface, our results yield the connectivity of the map

$$
\operatorname{Hom}\left(\pi_{1} \Sigma, U(n)\right)_{E}=\mathcal{A}_{\text {flat }}(E) / \mathcal{G}_{0}(E) \simeq \mathcal{A}_{\text {flat }}(E)_{h \mathcal{G}_{0}(E)} \rightarrow B \mathcal{G}_{0}(E),
$$

where the left-hand side denotes the homomorphisms whose associated bundle is isomorphic to $E$.

These results rely on an interesting combinatorial study of the Yang-Mills stratification. In particular, our connectivity formulas arise from a determination of the minimum non-zero Morse index of a Yang-Mills critical set, which is closely related to the connectivity of the space $\mathcal{C}_{\min }(E)$ (see Propositions 3.13 and 3.14). In the orientable case, weaker bounds on this quantity have been used in the literature before (see Daskalopoulos [5, Section 7], Daskalopoulos and Uhlenbeck [6], Ramras [14, and Cohen-Galatius-Kitchloo [4), but our precise formulas are new.

In Section 2 we discuss the necessary properties of the Yang-Mills stratification. The results in this section are essentially due to Atiyah and Bott and play a key role in the equivariant cohomology computations in [1. We feel that there is some value in filling in the details. In Section 3 we compute the minimum codimension, i.e. Morse index, of a non-central stratum and obtain the connectivity calculations described above.

\section{ThE HARDER-NARASIMHAN STRATIFICATION}

In this section we discuss the Harder-Narasimhan stratification on the space of holomorphic structures on a smooth, Hermitian vector bundle over a Riemann surface $M=M^{g}$, as in Atiyah-Bott [1, Section 7]. Recall that on a Hermitian bundle over a surface, the spaces of holomorphic structures and Hermitian connections are isomorphic. Under this isomorphism, the Harder-Narasimhan stratification agrees with the Morse stratification for the Yang-Mills functional, in the sense that 
the Yang-Mills flow defines deformation retractions from each Harder-Narasimhan stratum to its subset of Yang-Mills critical points [5, 13. We will use the phrases Harder-Narasimhan strata, Yang-Mills strata, and Morse strata more or less interchangeably in the orientable context, and we use the latter two terms in the non-orientable context.

Let $\mathcal{C}(E)=\mathcal{C}(n, k)$ denote the space of holomorphic structures on a rank $n$ Hermitian bundle $E \rightarrow M$ with Chern number $k$. As shown in 1, Sections 5, 7], this is an affine space, isomorphic to the affine space $\mathcal{A}(E)$ of Hermitian connections on $E$. As such, we may equip these spaces with Sobolev norms and complete them to Banach spaces. Throughout this paper, $\mathcal{C}(n, k)$ and $\mathcal{A}(E)$ will denote such Banach space completions (we will not need to specify the Sobolev regularity). Recall that the unitary gauge group $\mathcal{G}(E)=\mathcal{G}(n, k)$ of unitary automorphisms of $E$ and the larger complex gauge group $\mathcal{G}^{\mathbb{C}}(E)=\mathcal{G}^{\mathbb{C}}(n, k)$ of all complex automorphisms of $E$ (or rather their Sobolev completions) both act on $\mathcal{C}(n, k)$ [1, Section 14].

Given $\mathcal{E} \in \mathcal{C}(n, k)$ there is a unique filtration (the Harder-Narasimhan filtration)

$$
0=\mathcal{E}_{0} \subset \mathcal{E}_{1} \subset \cdots \subset \mathcal{E}_{r}=\mathcal{E}
$$

of $\mathcal{E}$ by holomorphic subbundles with the property that each quotient $\mathcal{D}_{i}=\mathcal{E}_{i} / \mathcal{E}_{i-1}$ is semi-stable $(i=1, \ldots, r)$ and $\mu\left(\mathcal{D}_{1}\right)>\mu\left(\mathcal{D}_{2}\right)>\cdots>\mu\left(\mathcal{D}_{r}\right)$, where the "slope" $\mu\left(D_{i}\right)$ is defined by $\mu\left(D_{i}\right)=\frac{\operatorname{deg}\left(D_{i}\right)}{\operatorname{rank}\left(D_{i}\right)}$. (A bundle $F$ is semi-stable if for all holomorphic subbundles $F^{\prime}<F, \mu\left(F^{\prime}\right) \leqslant \mu(F)$.) Letting $n_{i}=\operatorname{rank}\left(D_{i}\right)$ and $k_{i}=\operatorname{deg}\left(D_{i}\right)$, we have $\sum k_{i}=k, \sum n_{i}=n$. We call the sequence

$$
\mu=\left(\left(n_{1}, k_{1}\right), \ldots,\left(n_{r}, k_{r}\right)\right)
$$

the type of $\mathcal{E}$. The semi-stable stratum has type $((n, k))$. Let $\mathcal{C}_{\mu}=\mathcal{C}_{\mu}(n, k) \subset$ $\mathcal{C}(n, k)$ denote the subset of all holomorphic structures complex gauge-equivalent to a smooth structure of type $\mu$ (by Atiyah-Bott [1, Section 14], every $\mathcal{G}^{\mathbb{C}}(n, k)$ orbit contains a unique isomorphism class of smooth structures). We now have the following result from [1, Section 7] (see also [5, Theorem B]).

Theorem 2.1. Let $\mu=\left(\left(n_{1}, k_{1}\right), \ldots,\left(n_{r}, k_{r}\right)\right) \in \mathcal{C}(n, k)$. Then the stratum $\mathcal{C}_{\mu}$ is a locally closed submanifold of $\mathcal{C}(n, k)$ with complex codimension given by

$$
c(\mu)=\sum_{i>j}\left(n_{i} k_{j}-n_{j} k_{i}+n_{i} n_{j}(g-1)\right) .
$$

Next, we recall the partial ordering on the strata $\mathcal{C}_{\mu}$.

Definition 2.2. A sequence $\left(\left(n_{1}, k_{1}\right), \ldots,\left(n_{r}, k_{r}\right)\right)$ is admissible of rank $n$ and degree $k$ if $n_{i}>0$ for each $i, \sum n_{i}=n, \sum k_{i}=k$, and $\frac{k_{1}}{n_{1}}>\cdots>\frac{k_{r}}{n_{r}}$. We denote the set of all admissible sequences of rank $n$ and degree $k$ by $\mathcal{I}(n, k)$.

The correspondence $\mu \leftrightarrow \mathcal{C}_{\mu}$ defines a bijection between $\mathcal{I}(n, k)$ and the HarderNarasimhan strata. Following Shatz [15], we associate to each $\mu \in \mathcal{I}(n, k)$ a convex, piecewise-linear path $P(\mu)$ in $\mathbb{R}^{2}$, starting at $(0,0)$ and ending at $(n, k)$, with vertices $\left(\sum_{j=1}^{i} n_{j}, \sum_{j=1}^{i} k_{j}\right)(i=1,2, \ldots, n)$. Convexity corresponds to the condition that the slopes decrease. This yields a bijection between $\mathcal{I}(n, k)$ and the set of convex, piecewise-linear paths from $(0,0)$ to $(n, k)$ which change slope only at points in $\mathbb{Z}^{2}$. For any $\lambda, \mu \in \mathcal{I}(n, k)$, we set $\lambda \geqslant \mu$ if $P(\lambda)$ lies above $P(\mu)$, in the sense that if we consider these paths as the graphs of functions $[0, n] \rightarrow \mathbb{R}$, then $P(\lambda)(x) \geqslant P(\mu)(x)$ for all $x$. This makes $\mathcal{I}(n, k)$ a partially ordered set. 
Remark 2.3. When $g=0$, Grothendieck's theorem states that every holomorphic bundle is a sum of line bundles. Hence in genus zero, the stratum corresponding to $\mu \in \mathcal{I}(n, k)$ may be empty. For this reason, we assume $g>0$ throughout this paper (and in the non-orientable case we do not consider $\mathbb{R} P^{2}$ ).

A key fact about the Harder-Narasimhan stratification is the following result. For completeness, we will fill in some details of the proof.

Proposition 2.4 (Atiyah-Bott). The partial ordering $\leqslant$ on $\mathcal{I}(n, k)$ can be refined to a linear ordering $\mu_{1} \prec \mu_{2} \prec \cdots$ such that for any $j, \bigcup_{i=1}^{j} \mathcal{C}_{\mu_{i}}$ is open in $\mathcal{C}(n, k)$.

Let $E \rightarrow M$ be a rank $n$ Hermitian bundle over a non-orientable surface, and let $\widetilde{E} \rightarrow \widetilde{M}$ denote the pullback of $E$ to the orientable double cover of $M$. Then $\widetilde{E}$ is trivial and connections on $E$ pull back to connections on $\widetilde{E}$, yielding an embedding $i: \mathcal{A}(E) \hookrightarrow \mathcal{A}(\widetilde{E})$. Ho and Liu [7] define the Yang-Mills strata of $\mathcal{A}(E)$ to be the subsets $\mathcal{A}(E) \cap \mathcal{C}_{\mu}$, for $\mu \in I(n, 0)$. Proposition 2.4 implies:

Corollary 2.5. For any Hermitian bundle $E$ on a non-orientable surface, the linear ordering $\prec$ on $\mathcal{A}(\widetilde{E})$ induces a linear ordering on the Yang-Mills strata of $\mathcal{A}(E)$ such that the union of any initial segment $\left\{S \mid S \prec S_{0}\right\}$ is open in $\mathcal{A}(E)$.

Remark 2.6. The intersections $\mathcal{A}(E) \cap \mathcal{C}_{\mu}$ are sometimes empty. See [7, Section 7.1] for a precise determination of the non-empty strata.

We need a finiteness property of $\mathcal{I}(n, k)$, noted by Atiyah-Bott [1, p. 567].

Lemma 2.7. If $I \subset \mathcal{I}(n, k)$ is a finite collection of admissible sequences, then there are finitely many $\leqslant-$ minimal elements in the complement $I^{c}=\mathcal{I}(n, k)-I$.

Proof. If $P$ is a minimal path in $I^{c}$, then either $P$ is the minimum path (the line from $(0,0)$ to $(n, k))$ or $P$ is a minimal cover of some $Q \in I$. Hence it suffices to show that each path in $I(n, k)$ has only finitely many minimal covers.

Fix $\mu=\left(\left(n_{1}, k_{1}\right), \ldots,\left(n_{r}, k_{r}\right)\right) \in I(n, k)$ and let $P=P(\mu)$. Define

$$
s_{1}(P)=\max \left\{k_{1} / n_{1}, 0\right\} ; \quad s_{r}(P)=\min \left\{k_{r} / n_{r}, 0\right\} .
$$

Let $Q=P(\nu)$ for some $\nu \in I(n, k), \nu \neq((n, k))$. Let $h(Q)=\left(h_{1}(Q), h_{2}(Q)\right)$ denote the right endpoint of the rightmost line segment in $Q$ with slope at least $\frac{k}{n}$. If $h_{2}(Q) \geqslant n\left(s_{1}(P)-s_{r}(P)\right)+\max \{k, 0\}+1$, one checks that the path $Q^{\prime}$ with vertices $(0,0),\left(h_{1}(Q), h_{2}(Q)-1\right)$ and $(n, k)$ lies in $\mathcal{I}(n, k)$, and we claim that

$$
P \leqslant Q^{\prime}<Q \text {, and if } r>2 \text {, then } P<Q^{\prime}<Q \text {. }
$$

Assuming (2), we finish the proof. If $Q$ is a minimal cover of $P$, then either $r>2$ and $h_{2}(Q) \leqslant n\left(s_{1}(P)-s_{r}(P)\right)+\max \{k, 0\}$ or $r=2$ and $Q$ lies in the finite set of convex paths with $Q^{\prime}=P$. In the former case, $Q$ lies below the line of slope $\frac{k}{n}$ passing though $h(Q)$. Since $h_{1}(Q) \leqslant n$, this restricts $Q$ to a finite region.

To prove (2), note that $Q^{\prime}<\left((0,0),\left(h_{1}(Q), h_{2}(Q)\right),(n, k)\right) \leqslant Q$, so we need only check that $P<Q^{\prime}$ when $r>2$. If not, then at some time $x=x_{0}$, the path $P$ lies above the path $Q^{\prime}$ (since $\left.r>2, P \neq Q^{\prime}\right)$. If $x_{0} \leqslant h_{1}(Q)$, then the initial slope of $P$ must be more than the initial slope of $Q^{\prime}$. Our assumption on $h_{2}(Q)$ and the fact that $s_{r}(P) \leqslant 0$ give

$$
s_{1}(P) \geqslant \frac{k_{1}}{n_{1}}>\frac{h_{2}(Q)-1}{h_{1}(Q)} \geqslant \frac{\left(n\left(s_{1}(P)-s_{r}(P)\right)+\max \{k, 0\}+1\right)-1}{n} \geqslant s_{1}(P),
$$


a contradiction. If $x_{0}>h_{1}(Q)$, then a similar argument (using the facts that $s_{1}(P) \geqslant 0$ and $\left.s_{r}(P) \leqslant 0\right)$ yields the contradiction $s_{r}(P)>s_{r}(P)$.

The final ingredient in the proof of Proposition 2.4 is the following result regarding the closures on the Harder-Narasimhan strata.

Proposition 2.8. Let $S \subset \mathcal{I}(n, k)$ be a collection of admissible sequences that is upwardly closed, in the sense that if $\mu>\mu^{\prime}$ and $\mu^{\prime} \in S$, then $\mu \in S$ as well. Then the set $\bigcup_{\mu \in S} \mathcal{C}_{\mu}$ is closed.

Atiyah-Bott [1, (7.8)] and Daskalopoulos [5, Proposition 2.12] only claim that

$$
\overline{\mathcal{C}_{\mu}} \subset \bigcup_{\mu^{\prime} \geqslant \mu} \mathcal{C}_{\mu^{\prime}}
$$

To prove the (strictly stronger) statement in Proposition 2.8. we apply another result of Atiyah-Bott [1, Section 8].

Proposition 2.9. If $\mu=\left(\left(n_{1}, k_{1}\right), \ldots,\left(n_{r}, k_{r}\right)\right) \in I(n, k)$, then for any $A \in \mathcal{C}_{\mu}$,

$$
\inf _{g \in \mathcal{G}^{\mathbb{C}}(n, k)} L(g \cdot A)=\sum_{i=1}^{r} \frac{k_{i}^{2}}{n_{i}}=: l(\mu),
$$

where $L$ denotes the Yang-Mills functional.

Proof of Proposition 2.8. By (4), we have $\bigcup_{\mu \in S} \mathcal{C}_{\mu}=\bigcup_{\mu \in S} \overline{\mathcal{C}_{\mu}}$. The union of a locally finite collection of closed sets is closed, so it suffices to show that $\left\{\overline{\mathcal{C}_{\mu}}\right\}_{\mu}$ is locally finite. We will check that for each $N>0$, only finitely many of the closures $\overline{\mathcal{C}_{\mu}}$ meet the open set $L^{-1}[0, N)$.

If $L(A)<N$ for some $A \in \overline{\mathcal{C}_{\mu}}$, then continuity of $L$ and Proposition 2.9 imply that $l(\mu)<N$. There are finitely many $\mu \in \mathcal{I}(n, k)$ with $l(\mu) \leqslant N$, because $l(\mu) \leqslant N$ implies that $P(\mu)$ lies under the line $y=\sqrt{N} x$.

Remark 2.10. Although we will not need this fact, we point out that the number $l(\mu)$ appearing in Proposition 2.9 is actually the (unique) critical value of the YangMills functional on the stratum $\mathcal{C}_{\mu}$. This follows from convergence of the YangMills flow (Råde 13) and the fact that the Morse strata agree with the HarderNarasimhan strata (Daskalopoulos [5]), together with discreteness of the critical values of $L$. As mentioned in Råde [13, Section 2], this last result follows from Uhlenbeck Compactness and [13, Proposition 7.2].

Proof of Proposition 2.4. We construct a linear ordering $\prec$ on $\mathcal{I}(n, k)$ by setting $T_{0}=\{((n, k))\}$ and inductively defining

$$
T_{l}=T_{l-1} \cup\left\{\mu \in \mathcal{I}(n, k) \mid \mu \text { is minimal in } \mathcal{I}(n, k) \backslash T_{l-1}\right\},
$$

where we choose any linear ordering $\prec$ on $T_{l}$ extending the ordering $\prec$ on $T_{l-1}$ and satisfying $\mu \prec \eta$ if $\mu \in T_{l-1}$ and $\eta \in T_{l} \backslash T_{l-1}$. The set $T=\bigcup_{l} T_{l}$ is linearly ordered by $\prec$ (by Lemma 2.7, each $T_{l}$ is finite), and if $\mu \leqslant \eta$, then $\mu \prec \eta$. For each $j$, the set $\bigcup_{i=1}^{j} \mathcal{C}_{\mu_{i}}$ is open because by Lemma 2.7 and Proposition 2.8, its complement is a finite union of closed sets.

We now check that $T=\mathcal{I}(n, k)$. If $\mathcal{I}(n, k) \backslash T$ is non-empty, then we may choose a $\leqslant$-minimal element $\mu$ from this poset. There are finitely many $\eta$ with $\eta<\mu$, so we may choose a $\leqslant$-maximal element $\eta_{0}$ from the finite set $T_{\mu}=T \cap\{\eta \mid \eta<\mu\}$ (note that $T_{\mu} \neq \emptyset$ since it contains the minimum sequence $\left.((n, k))\right)$. Now, $\eta_{0} \in T_{N}$ 
for some $N$. If $\mu$ were a minimal cover of $\eta_{0}$ in $(\mathcal{I}(n, k), \leqslant)$, then by definition we would have $\mu \in T_{N+1}$, a contradiction. So we may choose a minimal cover $\eta_{1}$ of $\eta_{0}$ with $\eta_{0}<\eta_{1}<\mu$. Then $\eta_{1} \in T_{N+1}$, so $\eta_{1} \in T_{\mu}$, contradicting maximality of $\eta_{0}$. Hence $\mathcal{I}(n, k) \backslash T$ must be empty.

\section{Connectivity of the space OF CENTRAL YANG-MiLls CONNECTIONS}

On a Hermitian bundle $E$ over a Riemann surface $M$, the central Yang-Mills connections form the minimum critical set $\mathcal{C}_{\min }(E)$ of the Yang-Mills functional. In this section, we give a precise formula for the connectivity of these spaces depending only on the genus of $M$ and the rank and Chern number of $E$ (Proposition 3.13). We obtain a similar result (Proposition 3.14) for the space $\mathcal{A}_{\text {flat }}(E)$ of flat connections on a bundle $E$ over a non-orientable surface. Considering the actions of the gauge groups on these spaces leads to our main result, Theorem 3.15. Note that if $E$ has rank 1, then $\mathcal{C}_{\min }(E) \simeq \mathcal{C}_{\mathrm{ss}}(E)=\mathcal{C}(E) \simeq *$, so we assume the rank of $E$ is more than 1 in this section.

The starting point for our calculations is a homological argument, which reduces the problem to a combinatorial question about the codimensions of the Yang-Mills strata. The following example shows that the partial ordering $\leqslant$ on these strata does not respect codimensions; this complicates the arguments.

Example 3.1. The stratum $((1,1),(5,1)) \in \mathcal{I}(6,2)$ has complex codimension $4+$ $5(g-1)$, but lies above $((2,1),(4,1))$, whose complex codimension is $2+8(g-1)$.

For the remainder of the section, $E$ will denote a Hermitian bundle over $M^{g}$ $(g>0)$ of rank $n$ and Chern number $k$. Let $\prec$ denote a linear ordering on the set of Yang-Mills strata of $\mathcal{A}(E)$, as in Proposition 2.4 we will denote the strata by $\mathcal{C}_{\mathrm{ss}}=\mathcal{C}_{\mu_{0}} \prec \mathcal{C}_{\mu_{1}} \prec \cdots$. Recall that a space $X$ is (precisely) $d-$-connected if its homotopy groups $\pi_{i}(X)$ vanish for $i \leqslant d$ (and $\pi_{d+1} X \neq 0$ ).

Lemma 3.2. Let $d=d(E)$ denote the minimum positive (real) codimension of a Harder-Narasimhan stratum in the space $\mathcal{C}(E)$ of holomorphic structures on $E$. Then the space $\mathcal{C}_{\min }(E)$ of central Yang-Mills connections on $E$ and its stable manifold $\mathcal{C}_{\mathrm{ss}}(E)$ are precisely $(d-2)$-connected.

Similarly, let $\Sigma$ be a non-orientable surface and let $E$ be a complex bundle over $\Sigma$. Let $d=d(E)$ denote the minimum positive (real) codimension of a stratum in the space of connections $\mathcal{A}(E)$. If $\mathcal{A}(E)$ contains no strata of codimension $d+1$, then $\mathcal{A}_{\text {flat }}(E)$ is precisely $(d-2)$-connected.

To prove Lemma 3.2 we need a finiteness property of $\mathcal{I}(n, k)$, observed by Atiyah and Bott [1, p. 569]. For completeness, we give a proof.

Lemma 3.3. For any $D \in \mathbb{N}$, the set $\{\mu \in \mathcal{I}(n, k) \mid c(\mu)<D\}$ is finite, where $c(\mu)$ denotes the complex codimension of the stratum $\mathcal{C}_{\mu}$.

Proof. Recall from Theorem 2.1 that $c(\mu)=\sum_{i>j}\left(n_{i} k_{j}-n_{j} k_{i}+n_{i} n_{j}(g-1)\right)$. Say $\mu=\left(\left(n_{1}, k_{1}\right), \ldots,\left(n_{r}, k_{r}\right)\right) \in \mathcal{I}(n, k)$ and $c(\mu)<D$. Since $\sum n_{i}=n$, there are finitely many choices for the positive integers $n_{i}$. Each term in the sum defining $c(\mu)$ is positive (see Theorem 2.1), so $k_{1} n_{i}-k_{i} n_{1}<D$ and $k_{i}>\frac{k_{1} n_{i}-D}{n_{1}}$ for each $i>1$. If $k \geqslant 0$, then $k_{1}>0$, so $\frac{k_{1} n_{i}-D}{n_{1}}>-D$, while if $k<0$, then $\frac{k_{1}}{n_{1}} \geqslant \frac{k}{n}$, so $\frac{k_{1} n_{i}-D}{n_{1}} \geqslant \frac{k}{n} n_{i}-D>k / n-D$. Since $\sum k_{i}=k$, this leaves finitely many possibilities for the integers $k_{i}$. 
We will need to apply the Thom isomorphism theorem a number of times. This depends on the existence of tubular neighborhoods for the Yang-Mills strata $\mathcal{C}_{\mu_{i}}$ inside the open sets $\mathcal{C}_{i}:=\bigcup_{i=1}^{j} \mathcal{C}_{\mu_{i}}$. Although the construction is by now more or less standard, some subtleties arise due to the fact that the sets $\mathcal{C}_{\mu_{i}}$ are not closed. Hence we outline the argument. Our basic reference for Banach manifolds is Lang [11.

Lemma 3.4. Let $Y$ be a smooth, metrizable Banach manifold, and let $X \subset Y$ be a locally closed submanifold of finite codimension. Then there is an open neighborhood $\tau(X)$ of $X$ inside $Y$ which is diffeomorphic to the normal bundle $N(X)$.

Proof. We follow Lang [11, Section IV.5] and Bredon [3, VI.2]. As shown in [11, Chapter III], there is a smooth direct sum decomposition $\left.T(Y)\right|_{X}=T(X) \oplus N(X)$. Using sprays, Lang constructs an exponential map exp : $\mathcal{D} \rightarrow Y$, where $\mathcal{D} \subset T(Y)$ is an open neighborhood of the zero section, and shows that exp restricts to a local diffeomorphism $\mathcal{D} \cap N(X) \rightarrow Y$ (this means that each $x \in X$ has an open neighborhood $U_{x} \subset N(X)$ on which exp is a diffeomorphism onto an open set in $Y)$.

We claim that there exists an open set $W \subset \mathcal{D} \cap N(X)$ with the property that $\{w \in W \mid \exp (w) \in X\}=W \cap X$. Since $U_{x} \cap X$ is open in $X$, we have $U_{x} \cap X=W_{x} \cap X$ for some open set $W_{x} \subset Y$. Now $W=\bigcup_{x}\left(U_{x} \cap \exp ^{-1}\left(W_{x}\right)\right)$ is the desired open set in $N(X)$. Bredon [3, Chapter VI, Lemma 2.3] now shows that there exists a smaller neighborhood $W^{\prime} \subset W$ on which exp is injective (Bredon assumes both $W$ and $Y$ are metric spaces, but uses only the fact that $Y$ is metric).

Now exp : $W^{\prime} \rightarrow \exp \left(W^{\prime}\right)$ is a diffeomorphism onto an open neighborhood of $X$ inside $Y$. Finally, Lang [11, Section VII.4] shows that the finite-dimensional vector bundle $N(X)$ can be "compressed" into the neighborhood $W^{\prime}$.

Remark 3.5. Lang [11, Section IV.5] assumes that $X$ is closed in $Y$. This is used in the construction of the neighborhood $W^{\prime}$ above. One must replace a family $\left\{U_{\alpha}\right\}_{\alpha}$ of open sets in $Y$ which covers $X$ by a subordinate family $\left\{V_{\beta}\right\}_{\beta}$ which still covers $X$ and for which $\left\{\overline{V_{\beta}}\right\}_{\beta}$ is locally finite. This can always be done if $X$ is closed and $Y$ admits partitions of unity (Lang's hypotheses) but also works if $Y$ is metrizable, because then the union of the $U_{\alpha}$ is also metrizable.

Proposition 3.6. There are Thom isomorphisms in homology,

$$
H_{*}\left(\mathcal{C}_{i}, \mathcal{C}_{i-1} ; \mathbb{Z} / 2\right) \cong H_{*-2 c\left(\mu_{i}\right)}\left(\mathcal{C}_{\mu_{i}} ; \mathbb{Z} / 2\right),
$$

and similarly in the case of a non-orientable surface.

Proof. In the orientable case, $\mathcal{A}(E)$ is an (affine) Banach manifold, as are the open subsets $\mathcal{C}_{i}$. Daskalopoulos [5] showed that $\mathcal{C}_{\mu_{i}}$ is a locally closed submanifold of finite codimension, so by Lemma 3.4. $\mathcal{C}_{\mu_{i}}$ has a tubular neighborhood $\tau_{i}$ in $\mathcal{C}_{i}$. The isomorphisms (5) come from excising the complement of $\tau_{i}$ in $\mathcal{C}_{i}$ and applying the Thom Isomorphism Theorem to the bundle $\tau_{i} \rightarrow \mathcal{C}_{\mu_{i}}$. When $\Sigma$ is a non-orientable surface, pulling back to the orientable double cover $\widetilde{\Sigma}$ yields an embedding $\mathcal{A}(E) \hookrightarrow \mathcal{A}(\widetilde{E})$. The image is the fixed point set of an involution $\tau$ induced by the deck transformation on $\widetilde{\Sigma}$ (see Ho-Liu [7]). Morse strata in $\mathcal{A}(\widetilde{E})$ are intersections of Morse strata in $\mathcal{A}^{k-1}(\widetilde{E})$ with $\mathcal{A}(E)$, hence are locally closed submanifolds of finite codimension, and we apply Lemma 3.4 to obtain tubular neighborhoods. 
Proof of Lemma 3.2. The proofs in the orientable and non-orientable cases are essentially identical, so we work in the orientable case (the extra hypothesis in the non-orientable case is automatically satisfied in the orientable case because there the codimensions are always even).

Using transversality, it was shown in [14, Section 4] that $\pi_{i} \mathcal{C}_{\mathrm{ss}}(E)=0$ for $i \leqslant d-2$ (that argument was stated for the case $k=0$, but works in general). We must show that $\pi_{d-1} \mathcal{C}_{\mathrm{ss}}(E)$ is non-zero. Since $\mathcal{C}_{\mathrm{ss}}=\mathcal{C}_{\mathrm{ss}}(E)$ is (at least) $(d-2)$-connected, it suffices, by the Hurewicz Theorem, to prove that $H_{d-1}\left(\mathcal{C}_{\mathrm{ss}} ; \mathbb{Z}\right) \neq 0$. In fact, we claim that it is enough to show that $H_{d-1}\left(\mathcal{C}_{\mathrm{ss}} ; \mathbb{Z} / 2 \mathbb{Z}\right) \neq 0$. The Hurewicz Theorem implies that $H_{i}\left(\mathcal{C}_{\mathrm{ss}} ; \mathbb{Z}\right)=0$ for $i<d-1$, and hence $\operatorname{Tor}\left(H_{d-2}\left(\mathcal{C}_{\mathrm{ss}} ; \mathbb{Z}\right), \mathbb{Z} / 2 \mathbb{Z}\right)=0$. By the Universal Coefficient Theorem, we now have $H_{d-1}\left(\mathcal{C}_{\mathrm{ss}} ; \mathbb{Z} / 2 \mathbb{Z}\right) \cong H_{d-1}\left(\mathcal{C}_{\mathrm{ss}} ; \mathbb{Z}\right) \otimes$ $\mathbb{Z} / 2 \mathbb{Z}$ (note that this holds even if $d-1=0$ ), so if $H_{d-1}\left(\mathcal{C}_{\mathrm{ss}} ; \mathbb{Z} / 2 \mathbb{Z}\right.$ ) is non-zero, then $H_{d-1}\left(\mathcal{C}_{\mathrm{ss}} ; \mathbb{Z}\right) \neq 0$ as well. From now on, all homology groups will (implicitly) be taken with $\mathbb{Z} / 2 \mathbb{Z}$-coefficients 1

Let $\mathcal{C}_{\mu_{m}}$ denote the first stratum in our ordering with $c(\mu)=d$. We claim that $H_{d-1}\left(\mathcal{C}_{\mathrm{ss}}\right) \cong H_{d-1}\left(\mathcal{C}_{m-1}\right)$. Since $\mathcal{C}_{\mu_{j}}$ has codimension greater than $d$ for $j=1, \ldots, m-1$, the long exact sequences of the pairs $\left(\mathcal{C}_{j}, \mathcal{C}_{j-1}\right)$, together with Proposition 3.6. provide isomorphisms

$$
H_{d-1}\left(\mathcal{C}_{\mathrm{ss}}\right)=H_{d-1}\left(\mathcal{C}_{0}\right) \cong H_{d-1}\left(\mathcal{C}_{1}\right) \cong \cdots \cong H_{d-1}\left(\mathcal{C}_{m-1}\right) \text {. }
$$

It now suffices to show that $H_{d-1}\left(\mathcal{C}_{m-1}\right) \neq 0$. We argue by contradiction. If $H_{d-1}\left(\mathcal{C}_{m-1}\right)=0$, then applying Proposition 3.6 to each of the pairs $\left(\mathcal{C}_{j}, \mathcal{C}_{j-1}\right)$, $j=m-1, \ldots$, one finds that $H_{d-1}\left(\mathcal{C}_{j}\right)=0$ for all $j$. Now, let $\mathcal{C}_{\mu_{l}}$ be the largest stratum in our ordering with codimension $d$. The long-exact sequence for the pair $\left(\mathcal{C}_{l}, \mathcal{C}_{l-1}\right)$ has the form

$$
\cdots \longrightarrow H_{d}\left(\mathcal{C}_{l}\right) \longrightarrow H_{d}\left(\mathcal{C}_{l}, \mathcal{C}_{l-1}\right) \longrightarrow H_{d-1}\left(\mathcal{C}_{l-1}\right)=0 .
$$

Since $\mathcal{C}_{\mu_{l}}$ has codimension $d$, Proposition 3.6 implies that the relative term is nonzero. Hence the left-hand term $H_{d}\left(\mathcal{C}_{l}\right)$ must be non-zero as well.

The remaining strata have codimension at least $d+2$, so by Proposition 3.6 the inclusions $\mathcal{C}_{l} \subset \mathcal{C}_{l+1} \subset \cdots$ give isomorphisms

$$
H_{d}\left(\mathcal{C}_{l}\right) \stackrel{\cong}{\longrightarrow} H_{d}\left(\mathcal{C}_{l+1}\right) \stackrel{\cong}{\longrightarrow} \ldots \stackrel{\cong}{\longrightarrow} H_{d}\left(\mathcal{C}_{j}\right)
$$

for every $j>l$. By Proposition 2.4, $H_{d}(\mathcal{C}(E)) \cong \operatorname{colim}_{j} H_{d}\left(\mathcal{C}_{j}\right)$, but by (6) the colimit is non-zero, contradicting the fact that $\mathcal{C}(E)$ is contractible.

The additional hypothesis in the non-orientable case is satisfied in almost all cases, as we will see. We now turn to the question of computing the minimum codimension of a non-central stratum. We need some definitions and lemmas regarding the codimension of the Harder-Narasimhan strata.

Lemma 3.7. For any $\mu=\left(\left(n_{1}, k_{1}\right), \ldots,\left(n_{r}, k_{r}\right)\right) \in \mathcal{I}(n, k)$ with $r>2$,

$$
c\left(\left(n_{1}, k_{1}\right), \ldots,\left(n_{r}, k_{r}\right)\right)>c\left(\left(\sum_{i=1}^{r-1} n_{i}, \sum_{i=1}^{r-1} k_{i}\right),\left(n_{r}, k_{r}\right)\right) .
$$

Hence any admissible sequence minimizing the function c must have length 2.

\footnotetext{
${ }^{1}$ We work mod 2 because in the non-orientable case, the normal bundles to the Yang-Mills strata are real vector bundles. For $g>1$, these bundles are orientable by Ho-Liu-Ramras [10], but when $g=1$ this is not known.
} 
For $a \in \mathbb{R}$, we let $\lceil a\rceil$ denote the smallest integer strictly greater than $a$ (so for $a \in \mathbb{Z}$, we set $\lceil a\rceil=a+1$ ). This convention will simplify our notation.

Definition 3.8. Let $\mu_{m}=\mu_{m}(n, k)=\left(\left(m,\left\lceil\frac{k m}{n}\right\rceil\right),\left(n-m, k-\left\lceil\frac{k m}{n}\right\rceil\right)\right)$. The line from $(0,0)$ to $(n, k)$ passes through $\left(m, \frac{k m}{n}\right)$, so $\mu_{m} \in \mathcal{I}(n, k)$ and we define

$$
\mathcal{I}^{\prime}(n, k)=\left\{\mu_{m}: 0<m<n\right\} \subset \mathcal{I}(n, k) .
$$

Lemma 3.9. If $\mu=((m, l),(n-m, k-l)) \in \mathcal{I}(n, k)$, then $c(\mu) \geqslant c\left(\mu_{m}\right)$, with equality only if $\mu=\mu_{m}$. Hence if $\mu \in \mathcal{I}(n, k)$ minimizes $c$, then $\mu \in \mathcal{I}^{\prime}(n, k)$.

Definition 3.10. For $\mu=\left(\left(n_{1}, k_{1}\right), \ldots,\left(n_{r}, k_{r}\right)\right) \in \mathcal{I}(n, k)$, we define

$$
c_{1}(\mu)=\sum_{i>j}\left(n_{i} k_{j}-n_{j} k_{i}\right) \text { and } c_{2}(\mu)=(g-1) \sum_{i>j} n_{i} n_{j},
$$

so that $c(\mu)=c_{1}(\mu)+c_{2}(\mu)$.

We now consider the values that the function $c_{1}$ may take on the set $\mathcal{I}^{\prime}(n, k)$.

Definition 3.11. Given an integer $r$ and a natural number $n$, we let $[r]_{n}$ denote the unique integer between 1 and $n$ satisfying $r \equiv[r]_{n}(\bmod n)$.

Lemma 3.12. For any $m \in\{1, \ldots, n-1\}$, we have $c_{1}\left(\mu_{m}\right)=[k m]_{n}$, and

$$
c_{1}\left(\mathcal{I}^{\prime}(n, k)\right)=\{\operatorname{gcd}(n, k), 2 \operatorname{gcd}(n, k), \ldots, n\}
$$

(unless $\operatorname{gcd}(n, k)=1$, in which case $n$ is not included in this set).

Proof. Let $\left\lceil\frac{k m}{n}\right\rceil=\frac{k m}{n}+\frac{\epsilon_{m}}{n}$. Then $\epsilon_{m}=n\left\lceil\frac{k m}{n}\right\rceil-k m \in \mathbb{Z}$, and $\epsilon_{m} \equiv-k m(\bmod n)$. Since $1 \leqslant \epsilon_{m} \leqslant n$, we have $\epsilon_{m}=[-k m]_{n}$. A short calculation shows that $c_{1}\left(\mu_{m}\right)=$ $\epsilon_{m}$. Now, consider the set $\{1, \ldots, n\}$ as a cyclic group under addition modulo $n$. Then $c_{1}\left(\mathcal{I}^{\prime}(n, k)\right)=\left\{[-k m]_{n}: m=1,2, \ldots, n-1\right\}$. Since $[-k m]_{n} \equiv m[-k]_{n}(\bmod$ $n), c_{1}\left(\mathcal{I}^{\prime}(n, k)\right)$ is the subgroup of $\{1, \ldots, n\}$ generated by $[-k]_{n}$ (possibly excluding the zero-element $n)$. $\operatorname{But} \operatorname{gcd}(n, k)$ is the minimal element of this subgroup, so the lemma follows.

Proposition 3.13. If $E \rightarrow M^{g}$ is a Hermitian bundle over a Riemann surface $(g>0)$, then the connectivity of $\mathcal{C}_{\min }(E)$ and $\mathcal{C}_{\mathrm{ss}}(E)$ is precisely

$$
d(E)-2= \begin{cases}2 \operatorname{gcd}(n, k)-2 & \text { if } g=1, \\ 2 \min \left([k]_{n},[-k]_{n}\right)+2(g-1)(n-1)-2 & \text { if } g>1 .\end{cases}
$$

Proof. By Lemma 3.9, $c$ is minimized by $c\left(\mu_{m}\right)$ for some $m$. For $g=1, c_{2}$ is identically zero, so the result follows from Lemmas 3.12 and 3.2 For $g>1$, we find that

$$
c\left(\mu_{m}\right)-c\left(\mu_{1}\right)=[-m k]_{n}-[-k]_{n}+(g-1)\left(m n-m^{2}-n+1\right) .
$$

When $n \geqslant 6$, we will show that if $m \leqslant n / 2$, then $c\left(\mu_{m}\right) \geqslant c\left(\mu_{1}\right)$, and if $m \geqslant n / 2$, then $c\left(\mu_{m}\right) \geqslant c\left(\mu_{n-1}\right)$. This proves the proposition for $n \geqslant 6$, and the cases $n<6$ can be checked by hand.

We may assume $n>2$. First, say $2 \leqslant m \leqslant n / 2$. The function $f_{n}(m)=$ $m n-m^{2}-n+1$ is increasing on $\left(-\infty, \frac{n}{2}\right)$, and $f_{n}(2) \geqslant 0$. So $f_{n}(m) \geqslant 0$ for $m \leqslant \frac{n}{2}$, and hence $(g-1) f_{n}(m) \geqslant f_{n}(m)$. Equation (7) now gives

$$
c\left(\mu_{m}\right)-c\left(\mu_{1}\right) \geqslant[-m k]_{n}-[-k]_{n}+m n-m^{2}-n+1 .
$$


We now work with a generic integer $r$ in place of $-k$. Since $0<[r]_{n} \leqslant n$, we have $\frac{l}{m} n<[r]_{n} \leqslant \frac{l+1}{m} n$ for some $l \in\{0,1, \ldots, m-1\}$. Now $0<m[r]_{n}-l n \leqslant n$, so

$$
[m r]_{n}=m[r]_{n}-\ln .
$$

Furthermore, since $[r]_{n}>\frac{l n}{m}, l \leqslant m-1$, and $m \leqslant n / 2$, (9) implies that

$$
\begin{aligned}
{[m r]_{n}-[r]_{n} } & =\left(m[r]_{n}-\ln \right)-[r]_{n}=(m-1)[r]_{n}-l n \\
& >(m-1) \frac{\ln }{m}-\ln =-\frac{\ln }{m} \geqslant-\frac{(m-1) n}{m}=-n+n / m \\
& \geqslant-n+\frac{n}{n / 2}=-n+2 .
\end{aligned}
$$

Combining (10) and (8) yields

$$
c\left(\mu_{m}\right)-c\left(\mu_{1}\right)>-n+2+m n-m^{2}-n+1=n(m-2)-m^{2}+3 .
$$

Since $h_{n}(m)=n(m-2)-m^{2}+3$ is increasing for $m<n / 2$, it follows that if $m \geqslant 3$, then

$$
c\left(\mu_{m}\right)-c\left(\mu_{1}\right)>h_{n}(3)=n-9+3 \geqslant 0
$$

(since $n \geqslant 6)$. Note that $h_{n}(2)=-1$, so for $m=2$ a different bound is needed.

When $m=2, l \in\{0,1\}$. Following (10), we find that $[2 r]_{n}-[r]_{n}>-\frac{l n}{2} \geqslant-\frac{n}{2}$. Combined with (8) this yields

$$
c\left(\mu_{2}\right)-c\left(\mu_{1}\right)>-n / 2+2 n-4-n+1=n / 2-3 \geqslant 0
$$

(for $n \geqslant 6$ ). Thus we have shown that $c\left(\mu_{m}\right) \geqslant c\left(\mu_{1}\right)$ for $2 \leqslant m \leqslant n / 2$.

The proof that $c\left(\mu_{n-m}\right) \geqslant c\left(\mu_{n-1}\right)$ for $2 \leqslant m \leqslant n / 2$ is similar.

When $g=1$ and $k \neq 0$, this result shows that the connectivity of the space of central Yang-Mills connections does not tend to infinity with $n$.

We now turn to the case of a complex vector bundle $E$ over a non-orientable surface $\Sigma$. Here the minimum critical set of the Yang-Mills functional is the space $\mathcal{A}_{\text {flat }}(E)$ of flat connections. A combinatorial argument (simpler than the previous one) allows us to calculate the connectivity of $\mathcal{A}_{\text {flat }}(E)$ in most cases.

Proposition 3.14. Let $E$ be a complex bundle of rank $n>1$ over a non-orientable surface $\Sigma$, and let $\widetilde{g}$ denote the genus of the orientable double cover $\widetilde{\Sigma}$. If $\widetilde{g} \geqslant 2$ and $n \geqslant 9$, then $\mathcal{A}_{\text {flat }}(E)$ is precisely $(2 n \widetilde{g}-3 \widetilde{g}-1)$-connected.

Proof. We show that the minimum codimension of a non-flat stratum in $\mathcal{A}(E)$ is precisely $2 n \widetilde{g}-3 \widetilde{g}+1$ and that any other non-flat stratum has codimension at least $2 n \widetilde{g}-3 \widetilde{g}+3$. The result then follows from Lemma 3.2 .

Recall that $\mathcal{A}(E)$ embeds as the set of fixed points of an involution on $\mathcal{A}(\widetilde{E})$, and the Yang-Mills strata in $\mathcal{A}(E)$ are the intersections of the Yang-Mills strata of $\mathcal{A}(\widetilde{E})$ with these fixed points. Strata in $\mathcal{A}(\widetilde{E})$ containing fixed points have type

$$
\mu=\left(\left(n_{1}, k_{1}\right), \ldots,\left(n_{r}, k_{r}\right),\left(n_{0}, 0\right),\left(n_{r},-k_{r}\right), \ldots,\left(n_{1},-k_{1}\right)\right),
$$

for some $k_{i}, n_{i}$ (not all such strata contain fixed points [7, Section 7.1]). We call such sequences symmetric. The set of fixed points lying inside a symmetric stratum $\mathcal{C}_{\mu}$, if non-empty, has real codimension $c(\mu)$ inside $\mathcal{A}(E)$.

In analogy with Lemma 3.7, one sees that with $\mu$ as in (12) and $r>1$,

$$
c(\mu) \geqslant c\left(\left(\sum n_{i}, \sum k_{i}\right),\left(n_{0}, 0\right),\left(\sum n_{i},-\sum k_{i}\right)\right)+2,
$$


and therefore if $\mu$ is a symmetric stratum minimizing $c$, it must have the form $\mu=\left(\left(n_{1}, k_{1}\right),\left(n_{0}, 0\right),\left(n_{1},-k_{1}\right)\right)$. Next, one checks that if $k_{1}>1$,

$$
c\left(\left(n_{1}, k_{1}\right),\left(n_{0}, 0\right),\left(n_{1},-k_{1}\right)\right) \geqslant c\left(\left(n_{1}, 1\right),\left(n_{0}, 0\right),\left(n_{1},-1\right)\right)+2,
$$

so the minimum codimension is achieved by $\mu_{i}=((i, 1),(n-2 i, 0),(i,-1))$ for some $i \leqslant \frac{n}{2}$. Now $c(i):=c\left(\mu_{i}\right)=2 n-2 i+\left(2 n i-3 i^{2}\right)(\widetilde{g}-1)$ is quadratic in $i$ with a maximum at $\frac{n-1 /(\widetilde{g}-1)}{3}$, and one checks that for $\widetilde{g}>1$,

$$
c(2) \geqslant c(1)+2 \text { for } n \geqslant 9 \text { and } c(\lfloor n / 2\rfloor) \geqslant c(n / 2) \geqslant c(1)+2 \text { for } n \geqslant 12 .
$$

Hence when $\widetilde{g}>1$ and $n \geqslant 12$, the $c(i)$ is minimized when $i=1$ (and $c(1)=$ $2 n \widetilde{g}-3 \widetilde{g}+1)$. The stratum $\mu_{1}=((1,1),(n-1,1),(1,-1)$ is in fact non-empty because the degree zero factor has dimension $n-1>0$ [7, Section 7.1]. Hence $c(1)$ gives the minimum positive codimension of a non-empty stratum. The cases $n=9,10,11$ can be checked by hand.

Our reduction to the strata $\mu_{i}$ works for $n<9$, so these cases may be computed by hand. In most cases, one still obtains the connectivity of $\mathcal{A}_{\text {flat }}(E)$ precisely. But when $n=5$ and $\widetilde{g}=2$ or 4 , there is a (non-empty) stratum of codimension one more than the minimum, so Lemma 3.2 does not apply.

The case $\widetilde{g}=1$, where $\Sigma=K$ is the Klein bottle, is different. Let $E^{+}$and $E^{-}$ denote the (unique) trivial and non-trivial bundles over $K$ of rank $n$, respectively. When $n$ is even, strata of the form $((n / 2, k),(n / 2,-k))$ in $\mathcal{A}(E)$ may be empty: each such stratum for the trivial bundle on the double cover $S^{1} \times S^{1}$ contains connections from either $E^{+}$or $E^{-}$, but not both. When $2(n / 2)+k+2=n+k+2$ is even, this stratum contains connections from $E^{+}$, and when $n+k+2$ is odd, it contains connections from $E^{-}$[7, Proposition 7.1]. The reductions in the proof of Proposition 3.14 show that for odd $n$, the minimum codimension of a non-empty stratum is $n+1$ and the connectivity of $\mathcal{A}_{\text {flat }}(E)$ is precisely $n-1$. If $n$ is even and $E=E^{-}$is the non-trivial bundle, one finds that the stratum $((n / 2,1),(n / 2,-1))$ is non-empty and again gives the minimum codimension, namely $n$. So $\mathcal{A}_{\text {flat }}\left(E^{-}\right)$is precisely $(n-2)$-connected. Finally, if $E=E^{+}$, then these reductions show that any nonempty stratum has codimension at least two more than either $((n / 2,2),(n / 2,-2))$ or $\mu_{n / 2-1}=((n / 2-1,1),(2,0),(n / 2-1,-1))$, both of which are non-empty. The minimum codimension of a non-empty stratum is thus $n+2$, and $\mathcal{A}_{\text {flat }}\left(E^{+}\right)$is precisely $n$-connected.

We now prove our main result. A map $X \rightarrow Y$ is $n$-connected if it induces an isomorphism on $\pi_{k}$ for $k<n$ and a surjection on $\pi_{n}$; it is precisely $n$-connected if it is $n$-connected but not $(n+1)$-connected. We have fibration sequences

$$
\begin{aligned}
\mathcal{C}_{\min }(E) & \longrightarrow \mathcal{C}_{\min }(E)_{h \mathcal{G}(E)} \stackrel{q}{\longrightarrow} \operatorname{Map}^{E}(M, B U(n))=B \mathcal{G}(E) \\
\text { and } \mathcal{C}_{\min }(E) & \longrightarrow \mathcal{C}_{\min }(E)_{h \mathcal{G}_{0}(E)} \stackrel{q_{0}}{\longrightarrow} \operatorname{Map}_{*}^{E}(M, B U(n))=B \mathcal{G}_{0}(E),
\end{aligned}
$$

where $\mathcal{G}_{0}$ denotes the based gauge group. Since the quotient map $\mathcal{C}_{\min }(E) \rightarrow$ $\mathcal{C}_{\text {min }}(E) / \mathcal{G}_{0}(E)$ is a principal bundle [12, we have weak equivalences (see, for example, Atiyah-Bott [1, Section 13])

$$
\mathcal{C}_{\min }(E)_{h \mathcal{G}_{0}(E)} \simeq \mathcal{C}_{\min }(E) / \mathcal{G}_{0}(E) \text { and } \mathcal{C}_{\min }(E)_{h \mathcal{G}} \simeq\left(\mathcal{C}_{\min }(E) / \mathcal{G}_{0}(E)\right)_{h U(n)} .
$$

When $\mathcal{C}_{\text {min }}(E)$ consists of flat connections (which is the case for any bundle over a non-orientable surface, and for the trivial bundle over an orientable surface), the quotient $\mathcal{C}_{\min }(E) / \mathcal{G}_{0}(E)$ is simply the space $\operatorname{Hom}\left(\pi_{1}, U(n)\right)_{E}$, consisting of unitary 
representations of the fundamental group with associated bundle $E$. (Note that in the orientable case, the bundle associated to a representation is always trivial.) See, for example, [14, Sections 3 and 5] for further discussion. The long exact sequences in homotopy associated to (13), together with Propositions 3.13 and 3.14, immediately yield the following result.

Theorem 3.15. Let $E$ be a complex vector bundle of rank $n$ over a surface $M$ and consider the maps

$$
\mathcal{C}_{\min }(E)_{h \mathcal{G}(n)} \stackrel{q}{\rightarrow} \operatorname{Map}^{E}(M, B U(n)) \text { and } \mathcal{C}_{\min }(E)_{h \mathcal{G}_{0}(n)} \stackrel{q_{0}}{\rightarrow} \operatorname{Map}_{*}^{E}(M, B U(n)) .
$$

If $M$ is a genus $g$ Riemann surface, and $E$ has degree $k$, then the maps $q$ and $q_{0}$ are precisely $(2 \operatorname{gcd}(n, k)-1)$-connected if $g=1$ and precisely $\left(2 \min \left([k]_{n},[-k]_{n}\right)+\right.$ $2(g-1)(n-1)-1)$-connected if $g>1$.

Similarly, if $M$ is non-orientable, $n \geq q$, and the genus $\widetilde{g}$ of its orientable double cover is at least 2 , then the maps $q$ and $q_{0}$ are precisely $(2 n \widetilde{g}-3 \widetilde{g})$-connected.

From the discussion after Proposition 3.14, one can obtain similar calculations in some further cases, including when $\widetilde{g}=1$.

In the orientable case, $H^{*}(B \mathcal{G}(E) ; \mathbb{Z})$ and $H^{*}\left(B \mathcal{G}_{0}(E) ; \mathbb{Z}\right)$ were computed in Atiyah-Bott [1, Section 2]. For non-orientable surfaces, rational cohomology may be computed similarly (see Ho-Liu [8, Section 2]), so Theorem 3.15 yields computations of $H_{\mathcal{G}(E)}^{*}\left(\mathcal{C}_{\min }(E)\right)$ and $H_{\mathcal{G}_{0}(E)}^{*}\left(\mathcal{C}_{\min }(E)\right)=H^{*}\left(\mathcal{C}_{\min }(E) / \mathcal{G}_{0}(E)\right)$ in low dimensions.

\section{ACKNOWLEDGEMENTS}

The author thanks G. Carlsson, C. Groft, G. Helleloid, and C.-C. Liu for helpful conversations, and N.-K. Ho for pointing out some misstatements in an earlier draft.

\section{REFERENCES}

[1] M. F. Atiyah and R. Bott. The Yang-Mills equations over Riemann surfaces. Philos. Trans. Roy. Soc. London Ser. A, 308(1505):523-615, 1983. MR702806 (85k:14006)

[2] T. Baird. Antiperfection of Yang-Mills Morse theory over a nonorientable surface in rank three, arXiv:0902.4581, 2009.

[3] Glen E. Bredon. Introduction to compact transformation groups. Pure and Applied Mathematics, Vol. 46. Academic Press, New York, 1972. MR0413144 (54:1265)

[4] Ralph L. Cohen, Søren Galatius, and Nitoo Kitchloo. Universal moduli spaces of surfaces with flat bundles and cobordism theory. Adv. Math. 221(4): 1227-1246, 2009. MR2518637 (2010e:58007)

[5] Georgios D. Daskalopoulos. The topology of the space of stable bundles on a compact Riemann surface. J. Differential Geom., 36(3):699-746, 1992. MR.1189501 (93i:58026)

[6] Georgios D. Daskalopoulos and Karen K. Uhlenbeck. An application of transversality to the topology of the moduli space of stable bundles. Topology, 34(1):203-215, 1995. MR.1308496 (96e:32017)

[7] Nan-Kuo Ho and Chiu-Chu Melissa Liu. Yang-Mills connections on nonorientable surfaces. Comm. Anal. Geom. 16(3):617-679, 2008. MR2429971 (2009k:53054)

[8] Nan-Kuo Ho and Chiu-Chu Melissa Liu. Yang-Mills connections on orientable and nonorientable surfaces. Mem. Amer. Math. Soc., 202(948):viii+98, 2009. MR2561624

[9] Nan-Kuo Ho and Chiu-Chu Melissa Liu. Anti-perfect Morse stratification. arXiv:0808.3974, 2009.

[10] Nan-Kuo Ho, Chiu-Chu Melissa Liu, and Daniel A. Ramras. Orientability in Yang-Mills theory over nonorientable surfaces. Comm. Anal. Geom., 17(5):903-954, 2009. MR2643735

[11] Serge Lang. Differential and Riemannian manifolds, volume 160 of Graduate Texts in Mathematics. Springer-Verlag, New York, third edition, 1995. MR1335233 (96d:53001) 
[12] P. K. Mitter and C.-M. Viallet. On the bundle of connections and the gauge orbit manifold in Yang-Mills theory. Comm. Math. Phys., 79(4):457-472, 1981. MR623962 (83f:81056)

[13] Johan Råde. On the Yang-Mills heat equation in two and three dimensions. J. Reine Angew. Math., 431:123-163, 1992. MR1179335 (94a:58041)

[14] Daniel A. Ramras. Yang-Mills theory over surfaces and the Atiyah-Segal theorem, Algebr. Geom. Topol., 8:2209-2251, 2008. MR2465739(2009k:55008)

[15] Stephen S. Shatz. The decomposition and specialization of algebraic families of vector bundles. Compositio Math., 35(2):163-187, 1977. MR0498573(58:16668)

[16] Karen K. Uhlenbeck. Connections with $L^{p}$ bounds on curvature. Comm. Math. Phys., 83(1):31-42, 1982. MR0648356 (83e:53035)

Department of Mathematical Sciences, New Mexico State Univerity, P.O. Box 30001, Department 3MB, Las Cruces, New Mexico 88003-8001 